\title{
$\begin{array}{ll}\text { Research Square } & \begin{array}{l}\text { Preprints are preliminary reports that have not undergone peer review. } \\ \text { They should not be considered conclusive, used to inform clinical practice, } \\ \text { or referenced by the media as validated information. }\end{array}\end{array}$
}

\section{The distinguishing Predictive Value of CD4, CD8, and C - reactive protein in the Prognosis of Schistosomal and Non-Schistosomal Colorectal Cancer}

\author{
Qilin Zhai ( $\nabla$ zhaiql@163.com ) \\ Qingpu Branch of Zhongshan Hospital, Fudan University \\ Hongyan Jing \\ Qingpu Branch of Zhongshan Hospital, Fudan University \\ Dacheng Bu \\ Qingpu Branch of Zhongshan Hospital, Fudan University \\ Jing Liu \\ Qingpu Branch of Zhongshan Hospital, Fudan University \\ Kui Lu \\ Qingpu Branch of Zhongshan Hospital, Fudan University \\ Jican Liu \\ Qingpu Branch of Zhongshan Hospital, Fudan University \\ Yanchao Xu \\ Qingpu Branch of Zhongshan Hospital, Fudan University \\ Ting Zhu \\ Qingpu Branch of Zhongshan Hospital, Fudan University \\ Yingyong Hou \\ Qingpu Branch of Zhongshan Hospital, Fudan University \\ Junxia Yao \\ Qingpu Branch of Zhongshan Hospital, Fudan University \\ Weixia Wang \\ Qingpu Branch of Zhongshan Hospital, Fudan University
}

\section{Research Article}

Keywords: CD4+ T cell, CD8+ T cell, C-reactive protein, Schistosomiasis, colorectal cancer, Prognosis

Posted Date: March 3rd, 2022

DOI: https://doi.org/10.21203/rs.3.rs-1401969/v1

License: (a) (7) This work is licensed under a Creative Commons Attribution 4.0 International License. Read Full License 


\begin{abstract}
Background: The comparison of inflammation related factors between SCRC and non-schistosomal colorectal cancer (NSCRC) was not reported in previous literatures, and the inflammation based prognostic systems of SCRC were also unknown.
\end{abstract}

Aim: To explore the different roles of tumor infiltrating lymphocytes (TILs) and CRP in SCRC and in NSCRC, providing a possible predictive system to evaluate outcomes and to improve the risk stratification for CRC patients, especially for patients with schistosomiasis.

Methods: $351 \mathrm{CRC}$ tumours were evaluated for density of CD4+, CD8+ T cells and CRP in intratumoural and stromal compartments by immunohistochemical using tissue microarray.

Results: There were no association between TILs and CRP and schistosomiasis. Multivariate analysis identified sCD4 ( $p=0.038)$, iCD8 ( $p=0.003)$, schistosomiasis $(p=0.045)$ as independent prognostic factors for overall survival $(0 S)$ in the whole cohort; and sCD4 ( $p=0.006)$ and iCD 8 ( $p=0.020)$ were independent prognostic factors for OS in the NSCRC and SCRC set, respectively. Besides, we found that there were no differences of TILs and CRP, which were distributed in different areas of tumor tissue, between CRC patients with and without schistosomiasis.

Conclusion: TILs in different tumor areas has distinguished predictive value in CRC patients with schistosomiasis and without schistosomiasis, reflecting variable tumor immunoenvironments in them.

\title{
Introduction
}

Schistosomiasis is a chronic parasitic disease caused by a trematode blood fluke of genus schistosoma[1]. Schistosomias is a widespread endemic disease found in 74 countries [2] and over 250 million people are infected worldwide[3]. Schistosomiasis is now becoming a cause for concern in Europe, especially in southern Europe, because of climate change as well as infected travelers who return from endemic areas[4]. Qingpu District of Shanghai was once an endemic area of schistosomiasis, and the local people were deeply affected by it. There are still a large number of schistosomiasis-associated CRC patients left over from history. And schistosomiasis-associated CRC patients could be observed in our daily work occasionally.

It was suggested that schistosomiasis significantly correlated with increased colon cancer mortality in China[5]. Ming-Chai et al’s study[6] revealed a similarity between chronic schistosomiasis and ulcerative colitis regarding predisposition to carcinoma in that pseudopolyposis, ectopic regenerating glands, epithelial proliferation and ulceration are common in both of them. They suggested a strong relation between Schistosoma japonicum and CRC. A similar conclusion was drawn by Yu et al[7] from their studies on different types of schistosomal egg polyps. All these studies suggested that shistosomiasis is a risk factor for CRC. However, some suggested that if there is an increase in the risk of colorectal cancer, it is small $[8,9]$. Thus, the relationship between schistosomiasis and inflammation and colorectal cancer were necessary to be unraveled. However, related work has rarely been reported previously.

It was known that tumor-infiltrating lymphocytes (TILs) reflect an active inflammatory tumor microenvironment. Several studies have been made to assess the prognostic significance of TILs in human cancers, and pronounced lymphocytic infiltration has been shown to be a prognostic parameter for better survival in CRC[10-12]. However, inflammation based prognostic systems for schistosomal colorectal cancer has never been reported in the literature. TILs are composed of various lymphocytes with diverse functions. CD4+, CD $8+$ are the most common lymphocytes. Of them, $C D 8^{+} T$ cells play a crucial role in protective immunity against many infectious pathogens and can eradicate malignant cells by releasing perforins and granzymes, which may contribute to tumour cell death[13]. CD 4 + T cells secreted immunoregulatory cytokines such as IFN- $\gamma$ and TNF that may induce cytolytic T cell responses in tumours. Creactive protein (CRP), an acute phase reactant, primarily stimulates the innate immune system by facilitating phagocytosis, but also modulates adaptive immunity[14, 15]. Serum CRP has been shown to have prognostic value in CRC[16, 17]. However, the prognostic value of intratumor CRP remains unknown, especially in CRC patients with schistosomiasis.

With these considerations, we evaluated CD4+, CD8 + and CRP in CRC by immunohistochemical and compared their different prognostic roles between CRC patients with and without schistosomiasis. These results may uncover the important role of CD4+, CD8 + and CRP in CRC and provide a possible predictive system to evaluate outcomes for patients with schistosomiasis.

\section{Materials And Methods}

\section{Patients and sample selection.}

In this retrospective study, 351 unselected CRC patients who received curative resection without preoperative chemotherapy at Qingpu Branch of Zhongshan Hospital affiliated to Fudan University, from January 2008 to August 2016 were included. Clinicopathological information of patients in this survey was collected from clinical records and pathology reports. The local Ethics Committee of Qingpu Branch of Zhongshan Hospital approved the protocol of this study, which was conducted in accordance with the Declaration of Helsinki. Written informed consent was obtained from all patients. The inclusion and exclusion criteria were as previously described [18].

\section{Tissue microarray construction}

Tissue microarrays (TMA) were constructed as previously described[19]. Hematoxylin and eosin (H\&E)-stained slides from tissue blocks had been reviewed for adequacy of the representative areas of interest with a high density of tumour cells. The corresponding regions were marked on archival formalin-fixed, paraffin-embedded (FFPE) tissue blocks. The representative areas ( $2 \mathrm{~mm}$ wide and $6 \mathrm{~mm}$ long) of the tumour were extracted and then vertically planted into 
the recipient block one by one according to the corresponding location. The planting surface was aggregated on the aggregation instrument. An array was constructed with a maximum of 40 cores.

\section{Immunohistochemical analysis}

Three- to 5- $\mu \mathrm{m}$ thick CRC tissues were consecutively cut, subsequently dewaxed and rehydrated through graded alcohols. Slides were immunohistochemically stained in Roche Ventana Benchmark XT automated slide stainer (Ventana Medical Systems, Roche, France) according to the manufacturer`s instructions. Monoclonal and polyclonal anti-human antibodies were used for identification of CD4+ T cells (anti-CD4, 4B12, Dako), CD8+ T cells (anti-CD8, Ab4055, Abcam), CRP (Abcam, Cambridge, Massachusetts, Ab 32412, rabbit monoclonal Y284).

\section{Pathological assessment of CD4 and CD8+ T cell density and C-reactive protein}

The TMA slides were scanned using a scanner system (PRECICE 500B) at 40xmagnification. For CD4 and CD8, the densities of positively stained cells were evaluated on whole section slides using an image analysis system (Image $\mathrm{J}$ software, USA ) (cells per square millimeter) (Fig. 1A-B). At least half of the core area was selected randomly, and the results of the calculated densities were extracted and put into an Excel file. Measurements were recorded as the mean number of positive cells per tissue unit in square millimetres as well as the number of positive cells among each 1 -mm ${ }^{2}$ tissue units.

The C-reactive protein (CRP) staining were located in the stromal cells and tum or al cells cytoplasm in a diffused manner (Fig. 3 A-C).

\section{Detection of schistosome ova and assessment of tumor budding}

Schistosome ova were observed in all of original HE stained formalin-fixed paraffin-embedded (FFPE) sections (usually 4-6 slides), which were examined at $10 \times$ and $40 \times$ magnification fields using a conventional light microscope by two pathologists who were blinded to the clinical data. The diagnosis of schistosomiasis was done by finding schistosome eggs in HE-stained slides (Sup.Fig.1)

Tumor budding was defined as the presence of dedifferentiated single cells or small clusters of up to 5 cells at the invasive front of CRC[20]. The assessment of tumor budding was conducted as previously described[18]. Briefly, the 10-HPF method was used[21], the invasive front is first scanned at low magnification ( $4 \times$ to $10 x$ ) to identify areas of highest budding density. Tumor buds are then counted under high magnification (40x), and the tumor budding count is reported. The evaluation of tumor budding was conducted by two pathologists who were blinded to the clinical data. Five tumor budding counts were used as breakthrough point. In brief, tumor bud counts greater than or equal to 5 were defined as the high group, otherwise as the low group.

\section{Statistical analysis}

The associations between CD4 and CD8 and clinicopathologic features were analyzed using the chi-square $\left(\chi^{2}\right)$ test. Time-to-event outcomes were defined from date of initially curative resection to date of last follow-up. Univariate analysis was based on the Cox proportional hazards regression model. A multivariate Cox forward stepwise regression model was used to detect independent predictors of survival. The survival curves were compared using KaplanMeier method and log-rank test. All tests were two sided, and a $P$-value of less than 0.05 was considered as statistical significance. Data were analyzed using SPSS version 22.0 software (SPSS Inc., Chicago, IL, USA).

\section{Results \\ Study population}

The clinicopathologic features of a total of 351 cases of CRC were summarized in Table 1. Briefly, the age of patients with schistosomiasis was dominantly older than that of patients without schistosomiasis. Unexpectedly, there was no significant difference in morphology between colorectal cancer associated with schistosomiasis and that of without schistosomiasis (Table 1). In the whole cohort, the median age was 69 years (range 33-91), with frequencies between women $39 \%$ (137 out of 351 ) and men $61 \%$ (214 out of 351 ). The location of the tumor in $27 \%$ of patients was rectum, in left colon was $33 \%$ and in right colon was $40 \%$. On the basis of the AJCC Staging Manual (seventh edition), $76 \%$ cases were histologically graded as well to moderately differentiated, and $24 \%$ were poorly differentiated. The most predominant histological type was adenocarcinoma (311 patients), mucinous and signet ring cell carcinoma were $11 \%$ (34 patients). Vessel and nerve involvement were identified in $122(35 \%)$ and 31 (1\%) tumors, respectively. Lymph node metastasis was identified in 144 (40\%) patients. Stage I-II cases accounted for 54\% (190 out of 351), while stage III-IVa cases were $46 \%$ (161 out of 351 ), respectively. Schistosoma eggs could be observed under microscope in almost $39 \%$ cases.

\section{Immunohistochemical Findings}

$\mathrm{CD}^{+} \mathrm{T}$ and $\mathrm{CD} 8^{+} \mathrm{T}$ cell were observed both in cancer stroma and within cancer epithelium (i.e., intraepithelial). Representative pictures of lymphocyte infiltration are shown in Fig.1A-B. The distributions of $C D 4^{+} T$ and $C D 8^{+} T$ cell density are shown in Table 2 and Fig.2. The optimum cutoff value of CD4 $4^{+} T$ and $\mathrm{CD}^{+} \mathrm{T}$ cell density were determined by X-tile program, which were $29 \mathrm{cell} / \mathrm{mm}^{2}$ for intraepithelial CD4 (iCD4) (Table 2 and Sup Fig.2A-C) and 145 cell/mm ${ }^{2}$ for stroma CD4 (sCD4) (Table 2 and Sup Fig.3A-C) and 77 cell/mm² for intraepithelial CD8 (iCD8) (Table 2 and Sup Fig.4A-C) and 645 cell/mm² for stroma CD8 
(sCD8) (Table 2 and Sup Fig.5A-C). Patients were divided into 2 groups for further analysis based on their respective cutoff values. The C-reactive protein (CRP) positive staining were located in the stromal cells and tum or al cells cytoplasm in a diffused manner (Fig. 3A-C). The CRP positive staining was defined as positive, whereas negative staining was defined as negative.

\section{Association of TILs density and CRP with clinicopathological features}

The relationship between TILs density and patient demographics is listed in Table 3. No significant correlations were observed between iCD4+T cell density and clinical characteristics, such as age, gender, TNM stage, tumor differentiation and so on ( $\mathrm{p} \otimes 0.05)$. There was also no correlation between sCD4+T cell density and other clinical characteristics except for tumor budding $(p=0.031)$, lymph node metastasis $(p=0.045)$ and TNM stage $(p=0.001)$. Furthermore, there were significant association between iCD8+T cell density and age $(p<0.001)$, tumor deposit $(p=0.032)$. In addition, there were significant association between $\mathrm{sCD} 8+\mathrm{T}$ cell density and tumor deposit $(\mathrm{p}=0.004)$. However, there were no significant association between TILs in different tumor area and shistosomiasis $(\mathrm{p} \mathbb{8}$ $0.05)$.

The association between sCRP and tCRP and clinical characteristic were listed in Supplementary Table 1. Results demonstrated that sCRP was inversely associated with tumor size $(p=0.020)$ and colonic perforation $(p=0.001)$. Besides, tCRP was also negatively associated with colonic perforation ( $p=0.001)$. Unexpectedly, there were no relationship between tCRP or SCRP and schistosomiasis as well as CD4+T cells and CD8+T cells.

\section{Univariate and multivariate regression analysis}

In the whole cohort, univariate Cox regression analysis identified clinical factors statistically significantly associated with 0 (Table 4$)$ were age ( $\mathrm{p}=0.010)$, gender $(p=0.008)$, pathological T stage $(p<0.001)$, Lymph node metastasis $(p<0.001)$, TNM stage $(p<0.001)$, tumor differentiation $(p<0.001)$, lymphovascular invasion ( $p<0.001)$, tumor deposit $(p<0.001)$, tumor budding $(p<0.001)$, schistosomiasis $(p=0.044), i C D 4(P=0.015), s C D 4(p=0.002)$, iCD8 $(p<0.001)$, whereas only gender $(p=0.009)$, pathology T stage $(p=0.035)$, TNM stage $(p<0.001)$, sCD4 $(p=0.045)$, iCD8 ( $p=0.0089)$, schistosomiasis $(p=0.045)$ and tumor deposit were identified as independent risk factors for OS (Fig.4A).

In the NSCRC set, univariate Cox regression analysis identified clinical factors statistically significantly associated with OS (Table 4) were gender ( $\mathrm{P}=0.017)$, pathological T stage $(p=0.001)$, Lymph node metastasis $(p<0.001)$, TNM stage $(p<0.001)$, tumor differentiation $(p=0.003)$, lymphovascular invasion $(p<0.001)$, tumor deposit $(p<0.001)$, tumor budding $(p<0.001), \operatorname{sCD} 4(p<0.001)$, and iCD $8(p<0.001)$ were risk factors for 0S. Variables demonstrating a significant effect on OS were included in the multivariate analysis. Gender $(p=0.023)$, pathology $T$ stage $(p=0.046), s C D 4(p=0.006)$, tumor budding ( $p=0.034)$, and tumor deposit $(\mathrm{p}<0.0001)$ were identified as independent prognostic factors that associated with OS in the this set (Fig.4B).

In SCRC set, univariate Cox regression analysis identified clinical factors statistically significantly associated with OS (Table 4) were lymph node metastasis ( $p$ $<0.001)$, TNM stage $(p<0.001)$, tumor deposit $(p<0.001)$, iCD8 $(p=0.001)$ and SCD8 $(p=0.040)$ were risk factors for OS, whereas only Lymph node metastasis $(p<0.001)$, iCD8 $(p=0.020)$ and tumor deposit $(p<0.001)$ were identified as independent prognostic factors that associated with 0 in is set (Fig.4C). Unexpectedly, there were no association between stromal CRP and tumoral CRP and OS in the whole cohort and subgroups.

\section{Kaplan-Meier Analysis of OS}

Kaplan-Meier analysis, which was based on the cutoff value of the density of CD4+T cells and CD8+T cells in different tumor areas, was conducted to assess the variable in overall survival (OS) among different groups. In the whole cohort, compared to the low-density group, patients in the SCD4 and iCD8 highdensity group experienced significantly higher OS ( $p=0.0020, p<0.001$; Fig.5A and 5B). In the NSCRC set, patients in the $S C D 4$ high-density group gained beneficial OS compared to patients in the low-density group $(p=0.0004$, Fig.5C). Furthermore, patients in the iCD 8 high-density also possessed favorable survival compared to patients in the low-density $(p=0.0008$, Fig.5D). There was no association between sCRP or tCRP and OS in any subgroups.

\section{Comparison of TILs density and positive stained CRP in CRC patients with and without schistosomiasis}

We next compared the density of CD4+ and CD8+ T cells in CRC patients with or without schistosomiasis (data was not shown). The distribution of CD4+ T cells densities in stromal or tumoral cells between NSCRC and SCRC groups were no significant differences. Similarly, there were also no significant differences in the distribution of CD8+ T cells densities in stromal or tumoral cells between the NSCRC and SCRC group. The positivity rate of stromal CRP was $22 \%$ and $30 \%$ in the NSCRC set and SCRC set, respectively. Besides, the positivity rate of tumoral CRP was $14 \%$ and $15 \%$ in the NSCRC set and SCRC set, respectively.

\section{Discussion}

Schistosomiasis lead to inflammatory changes in CRC patients is well studied. However, direct evidences supporting inflammatory changes caused by inflammatory response after schistosomiasis infection is still lacking. Moreover, inflammation based prognostic systems for CRC were pronounced, but related predictors for schistosomal CRC have never been reported previously. In the present study, we found that CD $4+$ and CD8 + T cells distributed in different tumor areas were correlated with less aggressive tumor characteristics, but CRP distributed in different tumor areas were associated with more aggressive tumor features. In addition, results suggested that iCD4, SCD8, schistosomiasis, gender, pathological T stage, TNM stage and tumor deposit were 
independent prognostic factors for OS in the whole cohort; In the NSCRC set, SCD4, tumor budding, gender, pathological T stage and tumor deposit were independent prognostic factors for OS; In the SCRC set, iCD8, lymph node metastasis and tumor deposit were independent prognostic factors for OS. Furthermore, we found that there were no differences of TILs densities and CRP positivity, which were distributed in different areas of tumor tissue, between CRC patients with and without schistosomiasis.

Our results demonstrated that iCD4 and SCD8 were associated with favorable OS for CRC patients. However, when patients were divided into NSCRC and SCRC set based on status of schistosomal infection, SCD4 and iCD8 were independent prognostic factor for NSCRC patients and for SCRC patients, respectively. These results remind us that TILs distributed in different tumor areas have distinguished biological behavior and function, and the immune microenviroment is different in NSCRC and SCRC patients. Meanwhile, the findings require us to stratify patients with schistosomiasis and might facilitate patient counseling and management. The findings also pave the way for customized chemotherapy in CRC patients with schistosomiasis and without schistosomiasis individually based on the infiltration of TILs, because CRC patients with high levels of TILs could have improved outcome with 5-fluorouracilbased chemotherapy.

CD8 + T cells are cytotoxic T lymphocytes that directly attack cancer cells and play a central role in anti-cancer immunity[22]. Previous studies revealed substantial evidence that the density of CD8 + TILs was associated with the favorable survival in patients with various types of cancer[22-24]. Consistent with previous reports, this study confirmed the usefulness of CD8 + T-cell densities as prognostic factors (Fig. 4 and Fig. 5). CD4 + T-cell density has been reported to be a unbeneficial prognostic factor in other types of cancers, e.g. lung, renal, prostate and breast cancer[25-28]. On the contrary, Taichi Kuwahara et al's study showed that $C D+T$ cell densities were associated with favorable clinical outcomoes[29]. This is consistent with our results. The reason for this discrepancy remains unclear, but it may be because the function of $C D 4+T$ cells within the tumour microenvironment-i.e. in immune response activation or immunosuppression-may differ depending on the cancer type. Besides, we found that CRP was not associated with clinical outcome. This may be because CRP is a good predictor for clinical outcomes in the acute phase[30-32], but it is not suitable for chronic infection. It was well known that schistosoma egg deposited in the intestine provoke immune response and lead to chronic inflammation, and then lead to shisotomiasis. So it was speculated that the densities of TILs in NSCRC and SCRC patients was different. However, our results showed that the densities of TILs and CRP expression levels in the two sets were similar. As mentioned above, it may be because patients at diagnosis were not in the acute stage, but in the chronic stage. In addition, this may be explained that patients with schistosomiasis are obviously older than patients without schistosomiasis (Table 1), and the immune response of the elderly is weaker than that of the young.

Our retrospective study had several limitations. First, we do recognized the limitation of utilizing a TMA approach to assess expression of a biomarker that may only be locally present in samples, raising the possibility of false negatives, which could possibly change the significance of TILs in CRC. Second, because all results were generated at a single institution, the uniformity of the results may be low. Further work will be needed to validate the present results in a larger cohort of multicenters. Besides, validation studies may be performed on whole tissue slides to optimize the selection of regions most suitable for TILs assessment. Thirdly, the diagnosis of schistosomiasis was done by finding schistosome eggs in HE-stained slides. This will lead to positive cases miss and to generate data bias. We tried to use other methods to detect schistosomiasis related indicators, such as imaging data and serological tests. However, imaging data was not completed and serum could not be collected as the specimen used in this study was years ago. At present, more comprehensive materials related to schistosomiasis were collecting and will be used to validate results from this study.

\section{Conclusion}

In present study, iCD4 + and SCD4 + T cell densities were independent beneficial prognostic factors for NSCRC patients and schistosomal-CRC (SCRC) patients, respectively. We believe that this study is the first to report the prognostic significance of TILs and CRP in schistosomiasis-associated CRC patients. Hence, the results remind us to stratified CRC patients based on the status of schistosomal infection.

\section{Abbreviations}

colorectal cancer=CRC; Schistosomal colorectal cancer=SCRC; Schistosomal colorectal cancer=NSCRC; tumour infiltrating lymphocytes=TILs; C-reactive protein=CRP; Tissue microarray=TMA; intratumoral $C D 4=i C D 4$; stromal $C D 4=s C D 4$; intratumoral $C D 8=i C D 8$; stromal $C D 8=s C D 8 ; 0$ verall survival =0S; hematoxylin and eosin =H\&EखFormalin fixed paraffin-embedded =FFPE;

\section{Declarations}

\section{Acknowledgements:}

None

\section{Conflict of interest:}

The authors declare that they have no competing interests.

\section{Ethics approval and consent to participate:}


This study was approved by the medical ethics committee of Fudan University, in accordance with the Helsinki Declaration of 1975 . Prior written informed consent was obtained from all patients.

\section{Consent for publication $\square$}

Written informed consent was obtained from each participant.

\section{Availability of data and materials:}

The datasets used and/or analyzed during the current study are available from the corresponding authors on reasonable request.

\section{Funding:}

This work is supported by China Shanghai Municipal Commission of Health and Family Planning(Grant No. 20194Y0162 and w2021-25)

\section{Authors' contributions:}

Wang WW and Zhai QL: project development, data analysis, manuscript writing/editing;

Jing HY: data collection, manuscript writing;

Bu DC, Liu J, Lu K, Liu JC, Xu YC, Zhu T, Hou YY and Yao JX: data analysis \& collection, manuscript editing.

\section{References}

1. Bergquist, N.R., Schistosomiasis: from risk assessment to control. Trends Parasitol, 2002. 18(7): p. 309-14.

2. Bergquist, N.R., Schistosomiasis: from risk assessment to control. Trends Parasitol, 2002. 18(7): p. 309-14.

3. McManus, D.P., et al., Correction to: Schistosomiasis-from immunopathology to vaccines. Semin Immunopathol, 2020. 42(3): p. 373-374.

4. Colley, D.G. and W.E. Secor, Immunology of human schistosomiasis. Parasite Immunol, 2014. 36(8): p. 347-57.

5. Guo, W., et al., Correlations of colon cancer mortality with dietary factors, serum markers, and schistosomiasis in China. Nutr Cancer, 1993. 20(1): p. 13-20.

6. Ming-Chai, C., et al., Evolution of colorectal cancer in schistsosomiasis: transitional mucosal changes adjacent to large intestinal carcinoma in colectomy specimens. Cancer, 1980. 46(7): p. 1661-75.

7. Yu, X.R., et al., Histological classification of schistosomal egg induced polyps of colon and their clinical significance. An analysis of 272 cases. Chin Med J (Engl), 1991. 104(1): p. 64-70.

8. Yu, X.R., et al., Histological classification of schistosomal egg induced polyps of colon and their clinical significance. An analysis of 272 cases. Chin Med J (Engl), 1991. 104(1): p. 64-70.

9. Ojo, O.S., W.O. Odesanmi and O.O. Akinola, The surgical pathology of colorectal carcinomas in Nigerians. Trop Gastroenterol, 1992. 13(2): p. 64-9.

10. Zhao, Y., et al., The prognostic value of tumor-infiltrating lymphocytes in colorectal cancer differs by anatomical subsite: a systematic review and metaanalysis. World J Surg Oncol, 2019. 17(1): p. 85.

11. Ohtani, H., Focus on TILs: prognostic significance of tumor infiltrating lymphocytes in human colorectal cancer. Cancer Immun, 2007.7 : p. 4.

12. Pages, F., J. Galon and W.H. Fridman, The essential role of the in situ immune reaction in human colorectal cancer. J Leukoc Biol, 2008. 84(4): p. 981-7.

13. Arens, R. and S.P. Schoenberger, Plasticity in programming of effector and memory CD8 T-cell formation. Immunol Rev, 2010. 235(1): p. 190-205.

14. Lamb, G.W., et al., The relationship between the local and systemic inflammatory responses and survival in patients undergoing resection for localized renal cancer. BJU Int, 2008. 102(6): p. 756-61.

15. Jabs, W.J., et al., Expression of C-reactive protein by renal cell carcinomas and unaffected surrounding renal tissue. Kidney Int, 2005. 68(5): p. 2103-10.

16. Okugawa, Y., et al., Lymphocyte-C-reactive Protein Ratio as Promising New Marker for Predicting Surgical and Oncological Outcomes in Colorectal Cancer. Ann Surg, 2020. 272(2): p. 342-351.

17. Zhou, B., et al., C-reactive protein, interleukin-6 and the risk of colorectal cancer: a meta-analysis. Cancer Causes Control, 2014. 25(10): p. $1397-405$.

18. Wang, W., et al., Comparison of non-schistosomal colorectal cancer and schistosomal colorectal cancer. World J Surg Oncol, $2020.18(1)$ : p. 149.

19. Pan, W., et al., The prognostic role of c-MYC amplification in schistosomiasis-associated colorectal cancer. Jpn J Clin Oncol, 2020. 50(4): p. 446-455.

20. Koelzer, V.H., I. Zlobec and A. Lugli, Tumor budding in colorectal cancer-ready for diagnostic practice? Hum Pathol, 2016. 47(1): p. 4-19.

21. Horcic, M., et al., Tumor budding score based on 10 high-power fields is a promising basis for a standardized prognostic scoring system in stage II colorectal cancer. Hum Pathol, 2013. 44(5): p. 697-705.

22. Fridman, W.H., et al., The immune contexture in human tumours: impact on clinical outcome. Nat Rev Cancer, 2012. $12(4)$ : p. 298-306.

23. Pages, F., et al., In situ cytotoxic and memory T cells predict outcome in patients with early-stage colorectal cancer. J Clin Oncol, 2009. $27(35)$ : p. 5944-51. 
24. Noble, F., et al., Tumour infiltrating lymphocytes correlate with improved survival in patients with oesophageal adenocarcinoma. Cancer Immunol Immunother, 2016. 65(6): p. 651-62.

25. Kinoshita, T., et al., Prognostic value of tumor-infiltrating lymphocytes differs depending on histological type and smoking habit in completely resected non-small-cell lung cancer. Ann Oncol, 2016. 27(11): p. 2117-2123.

26. McArdle, P.A., et al., The relationship between T-lymphocyte subset infiltration and survival in patients with prostate cancer. Br J Cancer, 2004. 91(3): $\mathrm{p}$. 541-3.

27. Bromwich, E.J., et al., The relationship between T-lymphocyte infiltration, stage, tumour grade and survival in patients undergoing curative surgery for renal cell cancer. Br J Cancer, 2003. 89(10): p. 1906-8.

28. Droeser, R., et al., Differential pattern and prognostic significance of CD4+, FOXP3+ and IL-17+ tumor infiltrating lymphocytes in ductal and lobular breast cancers. BMC Cancer, 2012. 12: p. 134.

29. Kuwahara, T., et al., Intratumoural-infiltrating CD 4 + and FOXP3 + T cells as strong positive predictive markers for the prognosis of resectable colorectal cancer. Br J Cancer, 2019. 121(8): p. 659-665.

30. Allin, K.H. and B.G. Nordestgaard, Elevated C-reactive protein in the diagnosis, prognosis, and cause of cancer. Crit Rev Clin Lab Sci, 2011. 48(4): p. 15570.

31. Okugawa, Y., et al., Lymphocyte-C-reactive Protein Ratio as Promising New Marker for Predicting Surgical and Oncological Outcomes in Colorectal Cancer. Ann Surg, 2020. 272(2): p. 342-351.

32. Pathak, S., et al., Is C-reactive protein useful in prognostication for colorectal cancer? A systematic review. Colorectal Dis, 2014. 16(10): p. 769-76.

\section{Tables}

\section{Table 1}

Clinicopathological characteristics of the CRC cohort 


\begin{tabular}{|c|c|c|c|c|c|c|}
\hline \multirow[t]{2}{*}{ Characteristics } & \multicolumn{2}{|c|}{$\begin{array}{l}\text { All patients } \\
(\mathrm{N}=351)\end{array}$} & \multicolumn{2}{|c|}{$\begin{array}{l}\text { Paitents with shistosomiasis } \\
(\mathrm{N}=137)\end{array}$} & \multicolumn{2}{|c|}{$\begin{array}{l}\text { Patients without schistosomiasis } \\
(\mathrm{N}=214)\end{array}$} \\
\hline & $\mathrm{N}$ & $\%$ & $\mathrm{~N}$ & $\%$ & $\mathrm{~N}$ & $\%$ \\
\hline Age(『60ys) & 268 & 76 & 132 & 96 & 136 & 64 \\
\hline Gender(Male) & 214 & 61 & 86 & 63 & 126 & 59 \\
\hline \multicolumn{7}{|l|}{ Tumor location } \\
\hline Rectum & 94 & 27 & 37 & 27 & 57 & 27 \\
\hline Left colon & 115 & 33 & 51 & 37 & 64 & 30 \\
\hline Right colon & 142 & 40 & 49 & 36 & 93 & 43 \\
\hline Tumor size $(\llbracket 5 \mathrm{~cm})$ & 174 & 50 & 68 & 50 & 106 & 50 \\
\hline \multicolumn{7}{|l|}{ Differentiation } \\
\hline Well to moderately diff. & 267 & 76 & 102 & 74 & 165 & 77 \\
\hline Poor diff. & 84 & 24 & 35 & 26 & 49 & 23 \\
\hline Lymphovascular invasion (Negative) & 122 & 35 & 90 & 66 & 136 & 64 \\
\hline Nervous invasion (Negative) & 31 & 1.0 & 125 & 91 & 194 & 91 \\
\hline Tumor deposit & 42 & 1.2 & 120 & 88 & 189 & 88 \\
\hline Colonic perforation (Yes) & 13 & 0.4 & 5 & 4 & 8 & 4 \\
\hline Tummor budding ( $₫ 5$ cells) & 219 & 62 & 99 & 72 & 156 & 73 \\
\hline Ulceration (No) & 149 & 42 & 77 & 56 & 125 & 58 \\
\hline \multicolumn{7}{|l|}{ Histological type } \\
\hline Adenocarcinoma & 311 & 89 & 119 & 87 & 189 & 88 \\
\hline Mucinous/SRCC & 40 & 11 & 18 & 13 & 25 & 12 \\
\hline \multicolumn{7}{|l|}{ Pathological T stage } \\
\hline T1-2 & 80 & 23 & 29 & 21 & 51 & 24 \\
\hline Т3-4 & 271 & 77 & 108 & 79 & 163 & 76 \\
\hline \multicolumn{7}{|l|}{ Lymph node metastasis } \\
\hline No & 207 & 60 & 81 & 59 & 126 & 59 \\
\hline Yes & 144 & 40 & 56 & 41 & 88 & 41 \\
\hline \multicolumn{7}{|l|}{ TNM stage } \\
\hline $1+\|$ & 190 & 54 & 76 & 55 & 116 & 54 \\
\hline III+ IV & 161 & 46 & 61 & 45 & 98 & 46 \\
\hline schistosomiasis & 137 & 39 & - & - & - & - \\
\hline
\end{tabular}

\section{Table 2}

Densities and cut-off values of the tumour infiltrating lymphocytes.

$\begin{array}{lllll} & \text { Density }\left(\text { cell } / \mathrm{mm}^{2}\right) & \text { Median value } & \text { Cutoff value } & \text { Low-density group(\%) } \\ \text { iCD4 } & 0-1857 & 74 & 29 & 31 \\ \text { SCD4 } & 0-944 & 203 & 145 & 38 \\ \text { iCD8 } & 0-2554 & 291 & 77 & 19 \\ \text { SCD8 } & 0-2803 & 462 & 645 & 68\end{array}$

Notice: X-tile analysis of overall survival (OS) was performed using patients' data collected from the pathological system of the Qingpu District Center for Disease Control and Prevention to determine the optimal cut-off value for iCD4, SCD4, iCD8, sCD8 density. The optimal cut-off value for iCD 4 density was 29.0, for SCD 4 was 145.0 , for iCD 8 was 77 and for SCD 8 was 645 cell/mm2. Patients were divided into two groups: low-density group and high-density group based on the cutoff value of TILs. 
Table 3

The association between clinicopathological characteristics and STILs

\begin{tabular}{|c|c|c|c|c|c|c|c|c|c|c|c|c|}
\hline \multirow[t]{2}{*}{ Characteristic } & & \multicolumn{2}{|l|}{ iCD4 } & \multirow{2}{*}{$\begin{array}{l}P \\
\text { value }\end{array}$} & \multicolumn{3}{|l|}{ sCD4 } & \multicolumn{2}{|l|}{$i C D 8$} & \multirow{2}{*}{$\begin{array}{l}P \\
\text { value }\end{array}$} & \multicolumn{2}{|l|}{$s C D 8$} \\
\hline & & $\begin{array}{l}\text { Low } \\
(\mathrm{N}=110)\end{array}$ & $\begin{array}{l}\text { High } \\
(\mathrm{N}=241)\end{array}$ & & $\begin{array}{l}\text { Low } \\
(\mathrm{N}=135)\end{array}$ & $\begin{array}{l}\text { High } \\
(\mathrm{N}=216)\end{array}$ & $\begin{array}{l}P \\
\text { value }\end{array}$ & $\begin{array}{l}\text { Low } \\
(\mathrm{N}=65)\end{array}$ & $\begin{array}{l}\text { High } \\
(\mathrm{N}=286)\end{array}$ & & $\begin{array}{l}\text { Low } \\
(\mathrm{N}=238)\end{array}$ & $\begin{array}{l}\text { High } \\
(\mathrm{N}=113)\end{array}$ \\
\hline Age(ヌ60ys) & & & & 0.223 & & & 0.520 & & & $\begin{array}{l}0.001 \\
0\end{array}$ & & \\
\hline & $\triangle 60$ & 21 & 62 & & 29 & 54 & & 5 & 78 & & 55 & 28 \\
\hline & $\geq 60$ & 89 & 179 & & 106 & 162 & & 60 & 208 & & 188 & 85 \\
\hline Gender & & & & 0.198 & & & 0.574 & & & 0.095 & & \\
\hline & Male & 72 & 140 & & 79 & 133 & & 45 & 167 & & 148 & 68 \\
\hline & Female & 38 & 101 & & 56 & 83 & & 20 & 119 & & 95 & 45 \\
\hline Tumor site & & & & 0.676 & & & 0.773 & & & 0.442 & & \\
\hline & Rectum & 29 & 65 & & 34 & 60 & & 16 & 78 & & 66 & 30 \\
\hline & Left colon & 33 & 82 & & 45 & 70 & & 18 & 97 & & 75 & 41 \\
\hline & Right colon & 48 & 94 & & 56 & 86 & & 31 & 111 & & 102 & 42 \\
\hline Tumor size & & & & 0.818 & & & 0.913 & & & 0.496 & & \\
\hline & $\nabla 5 \mathrm{~cm}$ & 56 & 118 & & 67 & 107 & & 29 & 145 & & 115 & 63 \\
\hline & $\geq 5 \mathrm{~cm}$ & 54 & 123 & & 68 & 108 & & 36 & 141 & & 128 & 50 \\
\hline Differentiation & & & & 0.998 & & & 0.699 & & & 0.337 & & \\
\hline & Well diff. & 84 & 183 & & 100 & 167 & & 47 & 220 & & 182 & 88 \\
\hline & Poor diff. & 25 & 58 & & 35 & 49 & & 18 & 66 & & 61 & 25 \\
\hline $\begin{array}{l}\text { Lymphovascular } \\
\text { invasion }\end{array}$ & & & & 0.091 & & & 0.169 & & & 0.316 & & \\
\hline & Negative & 63 & 163 & & 80 & 146 & & 39 & 187 & & 158 & 70 \\
\hline & Positive & 47 & 78 & & 55 & 70 & & 26 & 99 & & 85 & 43 \\
\hline $\begin{array}{l}\text { Nervous } \\
\text { invasion }\end{array}$ & & & & 0.693 & & & 0.706 & & & 0.998 & & \\
\hline & Negative & 99 & 220 & & 124 & 195 & & 59 & 260 & & 219 & 104 \\
\hline & Positive & 11 & 21 & & 11 & 21 & & 6 & 26 & & 24 & 9 \\
\hline Tumor deposit & & & & 0.375 & & & 0.181 & & & 0.032 & & \\
\hline & $\leq 2$ & 94 & 215 & & 115 & 194 & & 52 & 257 & & 206 & 106 \\
\hline & $凶 2$ & 16 & 26 & & 20 & 22 & & 13 & 29 & & 37 & 7 \\
\hline $\begin{array}{l}\text { Colonic } \\
\text { perforation }\end{array}$ & & & & 0.555 & & & 0.259 & & & 0.715 & & \\
\hline & No & 105 & 233 & & 128 & 210 & & 62 & 276 & & 233 & 110 \\
\hline & Yes & 5 & 8 & & 7 & 6 & & 3 & 10 & & 10 & 3 \\
\hline Ulceration & & & & 0.163 & & & 0.739 & & & 0.998 & & \\
\hline & No & 57 & 145 & & 80 & 122 & & 37 & 165 & & 141 & 65 \\
\hline & Yes & 53 & 96 & & 55 & 94 & & 28 & 121 & & 102 & 48 \\
\hline Pathological T & & & & 0891 & & & 0.432 & & & 0.871 & & \\
\hline & I+ II & 24 & 56 & & 27 & 53 & & 14 & 66 & & 50 & 32 \\
\hline & III & 86 & 185 & & 108 & 163 & & 51 & 220 & & 193 & 81 \\
\hline Lymph node & & & & 0.198 & & & 0.045 & & & 0.677 & & \\
\hline & No & 59 & 148 & & 70 & 137 & & 37 & 170 & & 140 & 68 \\
\hline
\end{tabular}




\begin{tabular}{|c|c|c|c|c|c|c|c|c|c|c|c|}
\hline & Yes & 51 & 93 & 65 & 79 & & 28 & 116 & & 103 & 45 \\
\hline \multirow[t]{3}{*}{ TNM stage } & & & & & & 0.001 & & & 0.275 & & \\
\hline & $I+I I$ & 54 & 138 & 58 & 134 & & 32 & 160 & & 129 & 65 \\
\hline & III+ IV & 56 & 103 & 77 & 82 & & 33 & 126 & & 114 & 48 \\
\hline \multirow[t]{3}{*}{ Tumor budding } & & & & & & 0.031 & & & 0.888 & & \\
\hline & $\triangle 5$ cells & 64 & 146 & 73 & 137 & & 39 & 171 & & 139 & 72 \\
\hline & $\geq 5$ cells & 46 & 95 & 62 & 79 & & 26 & 115 & & 104 & 41 \\
\hline \multirow{3}{*}{$\begin{array}{l}\text { Histological } \\
\text { type }\end{array}$} & & & & & & 0.863 & & & 0.831 & & \\
\hline & Adenocarcinoma & 94 & 214 & 117 & 191 & & 57 & 251 & & 208 & 104 \\
\hline & Mucinous/SRCC & 16 & 27 & 18 & 25 & & 8 & 35 & & 35 & 9 \\
\hline \multirow[t]{3}{*}{ Schistosomiasis } & & & & & & 0.144 & & & 0.998 & & \\
\hline & Negtive & 65 & 149 & 75 & 139 & & 40 & 174 & & 147 & 67 \\
\hline & Positive & 45 & 92 & 59 & 78 & & 26 & 111 & & 96 & 41 \\
\hline
\end{tabular}

- Data is not applicable; Abbreviation: $N=$ Number; $L N=$ Lymph node; iCD4=intratumoral CD4; sCD4=stromal CD4; iCD8=intratumoral CD8; sCD8=stromal CD association

between schistosomiasis and clinicopathological characteristics was evaluated by using the Chi square and Fisher's exact tests. 
Table 4

Univariate and multivariate Cox regression of clinicopathological for overall survival

\begin{tabular}{|c|c|c|c|c|c|c|}
\hline \multirow[t]{2}{*}{ Variable } & \multicolumn{2}{|c|}{ All patients } & \multicolumn{2}{|l|}{ NSCRC } & \multicolumn{2}{|l|}{ SCRC } \\
\hline & $\mathrm{P}$ & $\mathrm{HR}(95 \% \mathrm{Cl})$ & $\mathrm{P}$ & $\mathrm{HR}(95 \% \mathrm{Cl})$ & $\mathrm{P}$ & $\mathrm{HR}(95 \% \mathrm{Cl})$ \\
\hline \multicolumn{7}{|l|}{ Univariate analysis } \\
\hline iCD 4 & 0.015 & $0.660(0.472-0.923)$ & 0.076 & $0.662(0.419-1.044)$ & 0.123 & $0.676(0.411-1.112)$ \\
\hline $\mathrm{sCD} 4$ & 0.002 & $0.602(0.435-0.834)$ & $\otimes 0.001$ & $0.452(0.292-0.701)$ & 0.750 & $0.924(0.566-1.507)$ \\
\hline iCD8 & $\varangle 0.001$ & $0.445(0.310-0.638)$ & 0.002 & $0.459(0.283-0.745)$ & 0.001 & $0.412(0.239-0.711)$ \\
\hline sCD8 & 0.176 & $0.776(0.538-1.120)$ & 0.057 & $0.612(0.369-1.015)$ & 0.040 & $0.538(0.297-0.972)$ \\
\hline sCRP & 0.761 & $1.060(0.728-1.545)$ & 0.478 & $1.231(0.685-2.210)$ & 0.640 & $1.168(0.610-2.234)$ \\
\hline tCRP & 0.263 & $0.754(0.460-1.236)$ & 0.796 & $0.906(0.430-1.908)$ & 0.301 & $0.615(0.244-1.547)$ \\
\hline Age ( $₫ 60 y s)$ & 0.010 & $1.759(1.142-2.708)$ & 0.122 & $1.454(0.905-2.336)$ & 0.232 & $21.827(0.139-3436.270)$ \\
\hline Gender (male/female) & 0.008 & $1.602(1.129-2.271)$ & 0.017 & $0.562(0.350-0.901)$ & 0.307 & $1.311(0.779-2.207)$ \\
\hline Tumor size(5cm) & 0.913 & $1.018(0.728-1.400)$ & 0.591 & $0.886(0.569-1.378)$ & 0.320 & $1.282(0.786-2.089)$ \\
\hline \multicolumn{7}{|l|}{ Tumor site } \\
\hline Rectum & & Refer & & Refer & & Refer \\
\hline Left colon & 0.908 & $1.025(0.676-1.553)$ & 0.672 & $0.889(0.515-1.534)$ & 0.484 & $1.263(0.657-2.427)$ \\
\hline Right colon & 0.464 & $0.859(0.572-1.290)$ & 0.054 & $0.590(0.344-1.010)$ & 0.130 & $1.631(0.865-3.076)$ \\
\hline Pathological T stage & $\varangle 0.001$ & 2.591(1.562-4.297) & 0.001 & $3.363(1.620-6.980)$ & 0.087 & $1.851(0.915-3.747)$ \\
\hline Lymph node metastasis & $\varangle 0.001$ & 2.802(2.012-3.902) & $\varangle 0.001$ & $2.447(1.573-3.807)$ & $\otimes 0.001$ & $3.552(2.141-5.894)$ \\
\hline TNM stage & $\otimes 0.001$ & $3.197(2.271-4.501)$ & $\otimes 0.001$ & $2.764(1.752-4.358)$ & $\otimes 0.001$ & $4.219(2.497-7.128)$ \\
\hline Differentiation & $\otimes 0.001$ & $1.889(1.334-2.674)$ & 0.003 & $2.009(1.259-3.206)$ & 0.054 & $1.668(0.991-2.809)$ \\
\hline Lymphovascular invasion & $\otimes 0.001$ & $3.251(1.987-5.318)$ & $₫ 0.001$ & $2.816(1.808-4385)$ & 0.275 & $1.321(0.801-2.180)$ \\
\hline Nervous invasion & 0.140 & $1.497(0.876-2.559)$ & 0.391 & $1.424(0.710-2.857)$ & 0.206 & $1.727(0.741-4.024)$ \\
\hline Tumor deposit & $\otimes 0.001$ & $4.006(2.686-5.973)$ & $\otimes 0.001$ & $3.973(2.359-6.692)$ & $\otimes 0.001$ & $4.138(2.205-7.769)$ \\
\hline Colonic perforation & 0.541 & $0.700(0.223-2.198)$ & 0.763 & $1.194(0.377-3.786)$ & 0.500 & $0.506(0.070-3.657)$ \\
\hline Tummor budding & $\otimes 0.001$ & $2.028(1.400-2.938)$ & $\varangle 0.001$ & $2.824(1.813-4.400)$ & 0.237 & $1.354(0.819-2.238)$ \\
\hline Schistosomiasis & 0.044 & $1.399(1.009-1.940)$ & - & - & - & - \\
\hline Ulceration & 0.624 & $0.9205(0.660-1.282)$ & 0.744 & $1.077(0.691-1.676)$ & 0.971 & $1.008(0.670-1.514)$ \\
\hline Histological type & 0.921 & $1.025(0.626-1.680)$ & 0.283 & $1.400(0.758-2.586)$ & 0.467 & $0.760(0.362-1.594)$ \\
\hline
\end{tabular}

- :Data is non-significant ; Abbreviation: $\mathrm{NSCRC}=$ patients without schistosomiasis; $\mathrm{SCRC}=$ patients with schistosomiasis; $\mathrm{Cl}=$ confidence interval; $\mathrm{HR}=$ Hazard ratio; LN= Lymph node;

iCD4=intratumoral $C D 4 ;$ SCD4=stromal $C D 4$; iCD8=intratumoral $C D 8$; $S C D 8=$ stromal $C D 8 ; P<0.05$ was defined as the criterion for variable deletion when performing backward stepwise selection.

\section{Figures}



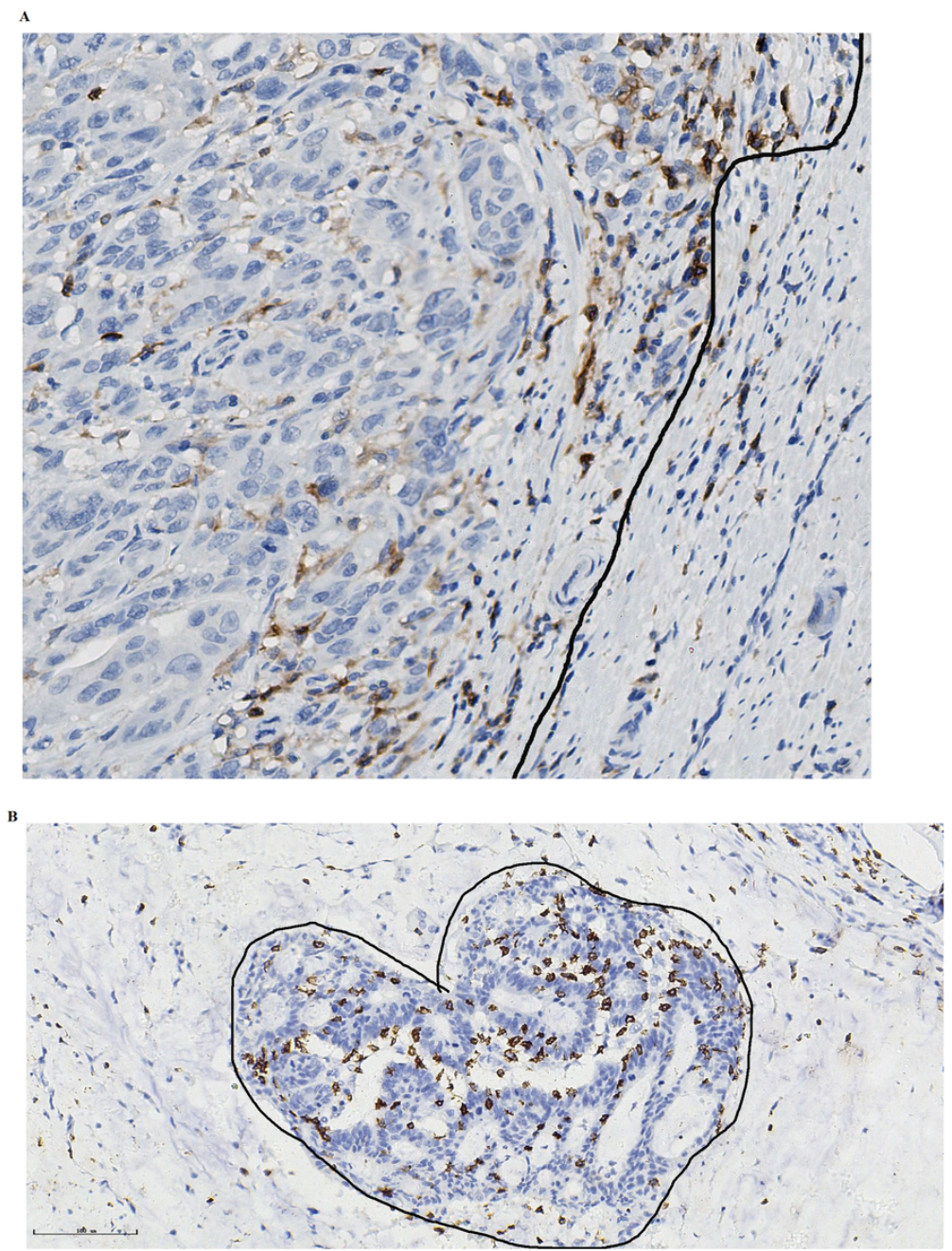

\section{Figure 1}

Immunohistochemical (IHC) staining of representative CD4 expression and CD8 expressionpositivity in different tumor areas. A. CD4 expression positivity $(100 \times)$ in the intratumoral area (iCD4) (inside the circle) and stromal area (sCD4) (outside the circle). B. CD8 expression positivity (200x) in the intratumoral area (iCD8) (inside the circle) and stromal area (sCD8) (outside the circle). 


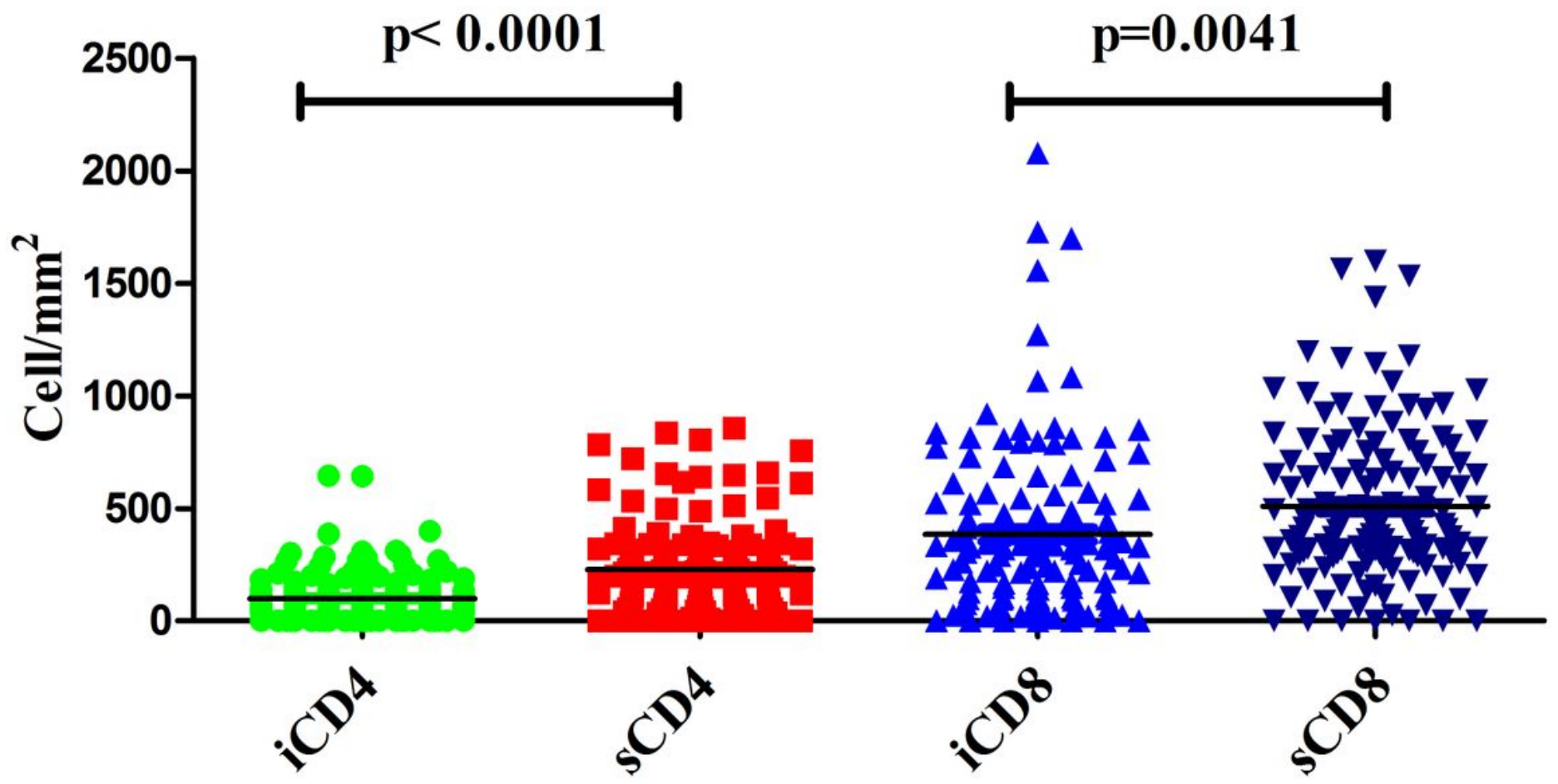

Figure 2

Heterogeneity of tumor-infiltrating immune cells. The density of iCD4+ (A), sCD4+ (B), iCD8+ (C), sCD8+ (E) cells differed significantly according to tumor location (results were examined by using the unpaired $t$-test).
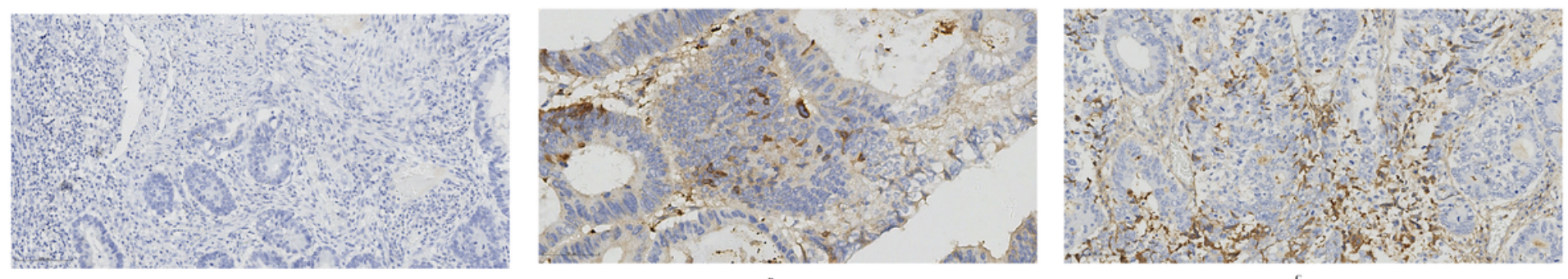

Figure 3

Immunohistochemical (IHC) staining pattern for C-reactive protein (CRP). A. a negative immunohistochemical staining pattern (100x); B. Positive IHC staining pattern for tumoral CRP(200x). C. Positive IHC staining pattern for stromal CRP(100x). 
A

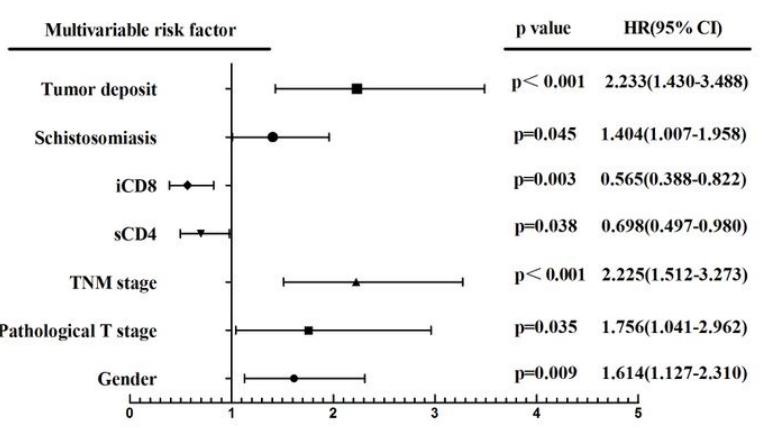

B

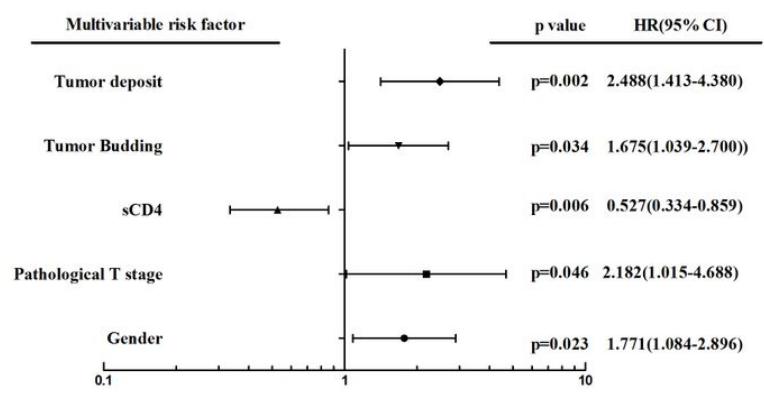

C

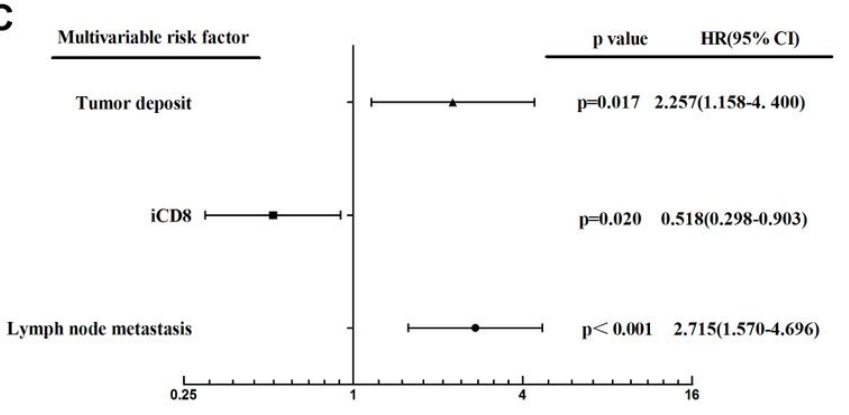

\section{Figure 4}

Prognostic analysis in CRC patients. A. Multivariate Cox regression analysis performed in the whole cohort; B. Multivariate Cox regression analysis performed in NSCRC patients, the value hazard ratio (HR) were log10 transformed; C. Multivariate Cox regression analysis performed in SCRC patients, the value hazard ratio were $\log 2$ transformed. 

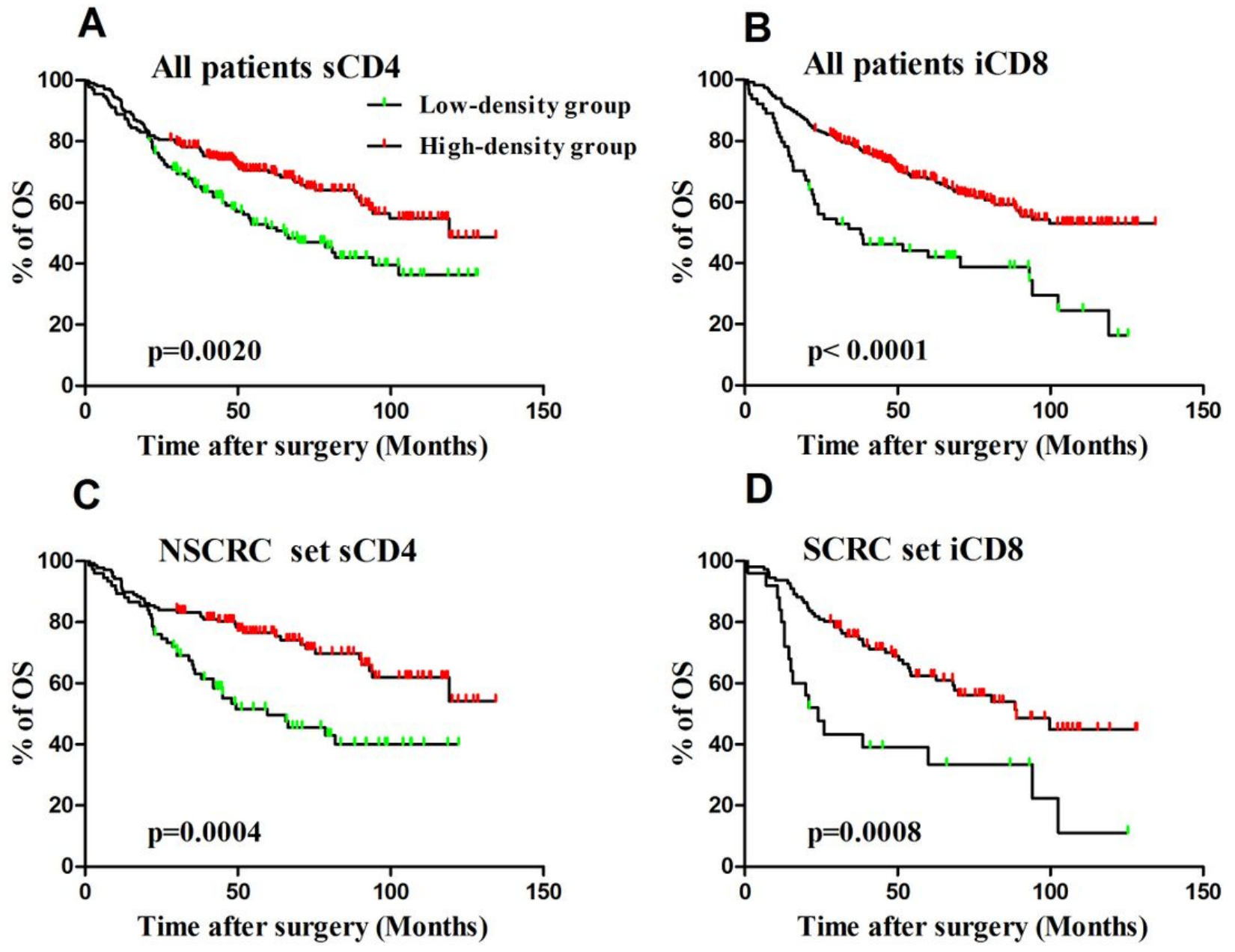

Figure 5

Kaplan-Meier curves for overall survival (OS) of colorectal cancer (CRC) patients with low or high TILs density. A. Stromal CD4+ T cell density in the whole cohort; B. Intratumoral CD8+ T cell density in the whole cohort; C. Stromal CD4+ T cell density in CRC patients without schistosomiasis (NSCRC); D. Intratumoral CD8+ T cell density in CRC patients with schistosomiasis (SCRC).

\section{Supplementary Files}

This is a list of supplementary files associated with this preprint. Click to download.

- 20210528SupMaterials.doc 